\title{
АНАЛИЗ РЕЗУЛЬТАТИВНОСТИ КОНТРОЛЬНЫХ МЕРОПРИЯТИЙ ПО КУРСУ «ОСНОВЫ СЕТЕВЫХ ТЕХНОЛОГИЙ»
}

\section{PERFORMANCE ANALYSIS \\ OF CONTROL MEASURES FOR THE COURSE "FUNDAMENTALS OF NETWORK TECHNOLOGIES" \\ S. Tulinov
E. Golubkova
A. Kruglov}

Summary: This article analyzes the effectiveness of control measures for the course "Fundamentals of Network Technologies" for RTU MIREA students, the charts for assessing the bank of questions implemented by teachers have been obtained. A method for transferring a bank of questions for students to a distance learning system has been developed. The article also proposes a solution that will allow students and teachers to rationalize and accelerate the defense of practical work in a distance learning environment. All of these solutions are aimed at reducing subjectivity while grading the students and increasing the number of students who reach the required level of understanding of the principles of construction and administration of computer networks.

Keywords: fundamentals of network technologies, practical work, valuation fund, Cisco NetAcad.

\author{
Тулинов Сергей Владимирович \\ Руководитель сетевой академии Сisco при РТУ МИРЭА \\ tulinov@mirea.ru \\ Голубкова Екатерина Михайловна \\ Инструктор сетевой академии Cisco при РТУ МИРЭА \\ golubkova@mirea.ru \\ Круглов Анатолий Михайлович \\ Инструктор сетевой академии Cisco при РТУ МИРЭА \\ kruglov@mirea.ru
}

Аннотация: В настоящей статье проведен анализ результативности контрольных мероприятий по курсу «0сновы сетевых технологий» для студентов РТУ МИРЭА, получены графики оценки реализованного преподавателями банка вопросов. Разработана методика перевода банка вопросов для студентов в систему дистанционного обучения.

В статье также предлагается решение, которое позволит студентам и преподавателям рационализировать и ускорить защиту практических работ в условиях дистанционного обучения. Оно направлено на уменьшение субъективизма при выставлении зачета по курсу и увеличение числа студентов, достигающих необходимого уровня понимания принципов построения и администрирования компьютерных сетей.

Ключевые слова: основы сетевых технологий, практическая работа, фонд оценочных средств, Cisco NetAcad.
$\mathrm{B}$ опросы совершенствования курсовых контрольных мероприятий постоянно находятся в центре внимания преподавателей. При этом в ходе получения высшего профессионального образования важна и внутренняя оценка результатов обучения - данная проблематика рассмотрена В [1, 2]. В [3] приводятся критерии качества разработанного фонда оценочных средств (ФОС) как основного механизма системы контроля образовательного процесса. В [4] акцент делается на описание методов и средств мониторинга процесса обучения и результата формирования у студентов профессиональных компетенций.

Спецификой курса «Основы сетевых технологий» является тот факт, что он построен на базе международной образовательной программы CCNA R\&S (Introduction to Networks и Routing and Switching Essentials) сетевой академии Cisco Netacad и позволяет студентам по окончанию обучения обрести компетенции сетевых технических специалистов и сетевых администраторов. Курс состоит из двух семестров, его подробная характеристика, два подхода к оценке знаний студентов при защите практических работ приводятся в статье авторов [5]. Первый подход традиционен и включает проверку вы- полненного задания по конфигурированию некоторой сетевой топологии, а также опрос по теоретическому материалу лекций из материалов на портале Cisco Netacad [6]. Однако дефицит бюджета времени на защиту той или иной работы может привести к субъективизму со стороны преподавателя, т.к. трудно учесть индивидуальные особенности студентов, на чем частично концентрируют внимания авторы в [7]. Второй метод направлен на дополнительное использование разработанного авторами ФОС в виде тестовых вопросов по всем темам курса и позволяет рационализировать бюджет времени общения студентов и преподавателей в аудиторном или дистанционном режимах.

В прошедшем учебном году были использованы оба подхода, следовательно, теперь необходимо получить обратную связь от студентов и выяснить, насколько результативными оказались новые контрольные мероприятия. Выявление эффективности предложенных контрольных мероприятий является основной задачей настоящей статьи. Для решения этой задачи был выбран метод анкетирования, составлены шесть вопросов с выбором одного варианта ответа и двух вопросов с развернутым ответом. Далее анкета была выборочно отправле- 
на некоторому количестве обучающихся. Для получения объективных мнений анкетирование проводилось при условиях добровольности, анонимности и до прохождения зачета. Перечень вопросов и варианты ответов представлены в таблице 1.

Таблица 1.

\section{Вопросы для внутренней оценки контрольных мероприятий}

\begin{tabular}{|c|c|c|}
\hline № & Вопрос & Варианты ответа \\
\hline 1 & $\begin{array}{l}\text { Сколько практических работ } \\
\text { Вы защитили в прошлом } \\
\text { семестре? }\end{array}$ & $\begin{array}{l}0 \\
1-3 \\
4-6 \\
7-9\end{array}$ \\
\hline 2 & $\begin{array}{l}\text { Сколько практических работ } \\
\text { Вы защитили в текущем } \\
\text { семестре? }\end{array}$ & $\begin{array}{l}0 \\
1-4 \\
5-8 \\
9-12\end{array}$ \\
\hline 3 & $\begin{array}{l}\text { В каком семестре оценка } \\
\text { знаний проходила наиболее } \\
\text { объективно? }\end{array}$ & $\begin{array}{l}\text { В предыдущем семестре. } \\
\text { В этом семестре }\end{array}$ \\
\hline 4 & $\begin{array}{l}\text { Считаете ли Вы правильным } \\
\text { введение индивидуализа- } \\
\text { ции для практических работ } \\
\text { (использование фамилии и } \\
\text { номера в группе)? }\end{array}$ & Шкала от 1 до 5 \\
\hline 5 & $\begin{array}{l}\text { Насколько хорошо были } \\
\text { составлены тестовые во- } \\
\text { просы? }\end{array}$ & Шкала от 1 до 5 \\
\hline 6 & $\begin{array}{l}\text { Помогли ли Вам тестовые } \\
\text { вопросы улучшить качество } \\
\text { знаний по курсу? }\end{array}$ & $\begin{array}{l}\text { Да, спомощью них я } \\
\text { смог(ла) углубить свои } \\
\text { знания. } \\
\text { Нет, я не смог(ла) получить } \\
\text { от них пользу. } \\
\text { В некоторой степени тесто- } \\
\text { вые вопросы помогли при } \\
\text { изучении курса, но меня } \\
\text { не устроили формулировки } \\
\text { вопросов }\end{array}$ \\
\hline 7 & $\begin{array}{l}\text { Что Вам не понравилось в } \\
\text { образовательном процессе? }\end{array}$ & Развернутый ответ \\
\hline 8 & $\begin{array}{l}\text { Что Вам понравилось в об- } \\
\text { разовательном процессе, и } \\
\text { как его можно улучшить? }\end{array}$ & Развернутый ответ \\
\hline
\end{tabular}

После проведения анкетирования для студентов 3 курса Института ИТ РТУ МИРЭА в конце 2 семестра было получено 136 ответов. На рисунке 1 представлено сравнение количества защищенных работ в 1 и 2 семестрах. Процент студентов, выполнивших и защитивших наибольшее число практических работ, увеличился, что видно из нижней диаграммы на рисунке 1. Следует учитывать тот факт, что успешные и сильно мотивированные студенты, уже работающие в сфере сетевых технологий, могут самостоятельно изучить курс без сдачи промежуточных заданий - для них выстраивается индивидуальная зачетная траектория. Таким образом, наблюдается положительная тенденция роста числа студентов, которые успешно изучили материал курса в полном объеме.

Необходимо также отметить, что почти 70\% респондентов посчитали, что новые методы оценивания являются более объективными, т.к. влияние субъективизма при защите работ с использованием тестирования значительно уменьшается.

Немаловажной задачей была оценка по 5-бальной шкале мнения студентов относительно введения индивидуализации в практические работы. Из гистограммы на рисунке 2 видно, что почти треть опрошенных посчитали такие нововведения правильными и поставили наивысший балл.

Важным критерием формирования ФОС является однозначность формулировок и полнота оценочных материалов по каждой практической работе, в связи с этим авторы попросили студентов высказать собственное мнение и оценить, насколько хорошо по 5-бальной шкале были составлены тестовые вопросы. Из гистограммы на рисунке 3 видно, что 79,4\% респондентов дали достаточно высокую оценку разработанным вопросам из ФОС (от 4 до 5 баллов). Тем не менее, большая распределенность ответов говорит о том, что необходимо пересмотреть формулировки некоторых вопросов, которые могли быть восприняты неоднозначно.

Кроме того, студентам предложили ответить на вопрос «Помогли ли Вам тестовые задания улучшить качество знаний по курсу?». Большинство из них, а именно 70,6\%, ответили положительно. 14,7\% высказали прямо противоположное мнение, и еще столько же студентов сошлись на мнении, что в некоторой степени тестовые вопросы помогли при изучении курса, но их не устроили формулировки вопросов.

В связи с данным обстоятельством, на очередном этапе разработки педагогических тестов авторами было решено произвести повторный анализ ФОС и коррекцию формулировок для некоторых тестовых заданий. Всего было модернизировано 14\% вопросов, которые могли быть восприняты неоднозначно.

Наиболее важной и полной оценкой студентов являлось развернутое суждение о достоинствах и недостатках контрольных мероприятий по курсу. Проанализировав все ответы, авторы выявили и обобщили следующие недостатки:

- отсутствие возможности прохождения тестов в режиме онлайн; 

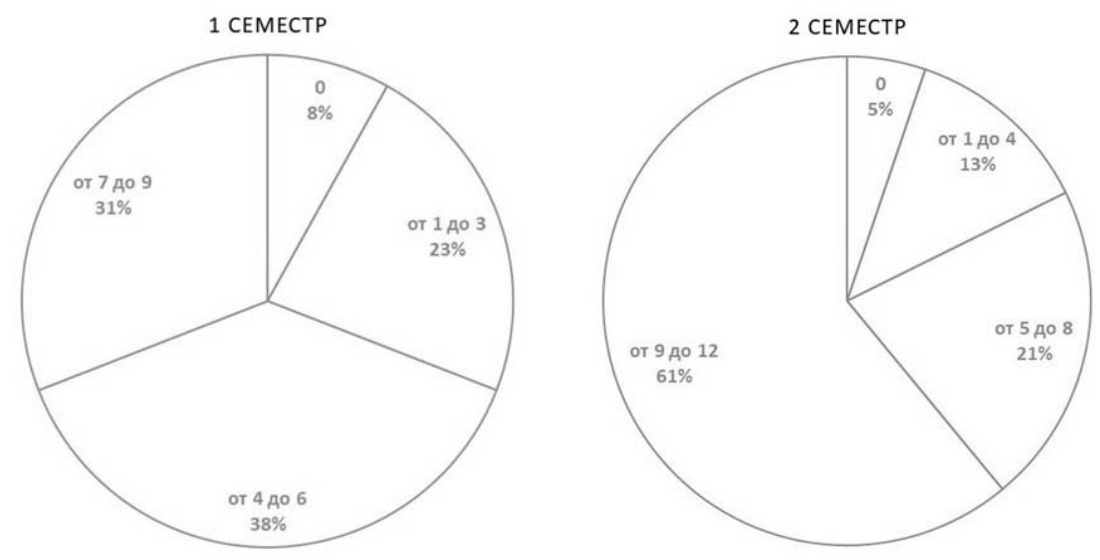

Рис. 1. Рост количества защищенных практических работ студентами [Получено авторами]

Гистограмма оценки введения индивидуализации практических работ

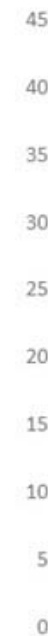

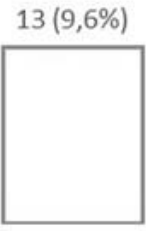

1

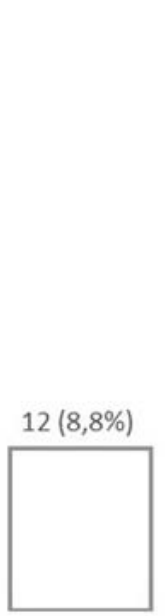

2

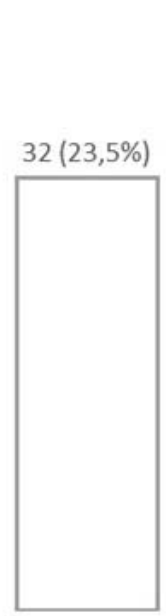

3

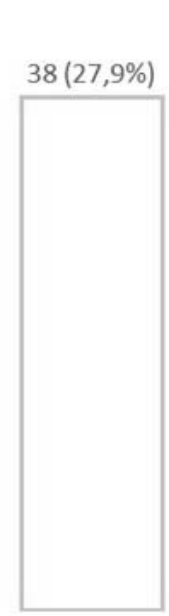

4
$41(30,1 \%)$

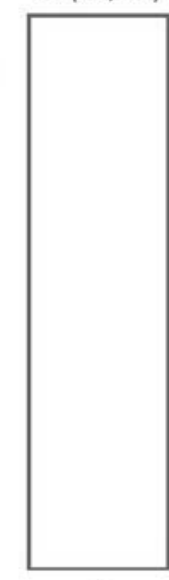

5

Рис. 2. Гистограмма оценки введения индивидуализации практических работ [Получено авторами]

Гистограмма оценки тестовых вопросов по практическим работам

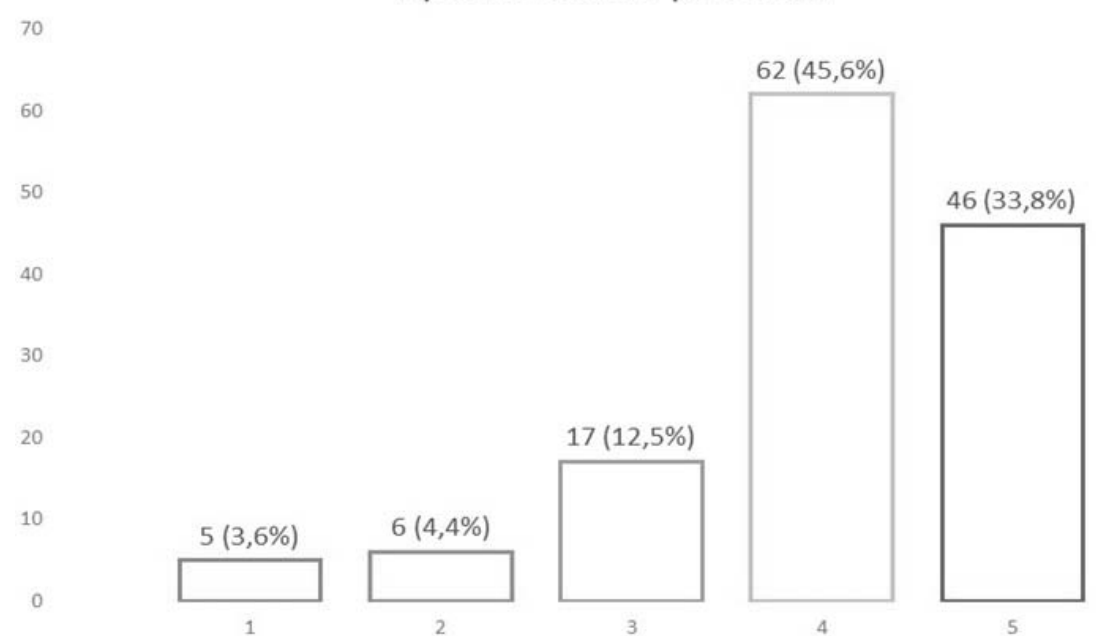

Рис. 3. Гистограмма оценки тестовых вопросов по практическим работам [Получено авторами] 
- единственная попытка сдачи практической работы в неделю;

- разброс вариантов по уровню сложности;

- ограниченность времени общения с преподавателем;

- не всегда понятная формулировка вопроса.

Все вышеперечисленные суждения являются основанием для доработки существующих контрольных мероприятий и будут учитываться в дальнейшем. Также студентам было предложено высказать свое мнение по поводу преимуществ новой системы оценки знаний. Наиболее часто встречающиеся мнения указаны ниже:

- защита работ проходила быстро и эффективно (26\%);

- введение тестов значительно сократило время защиты и повысило объективность оценивания знаний для всех студентов (32\%);

- защищенные работы дают мотивационные бонусы при написании зачета, который включает в себя комплексную практическую работу по настройке некоторой сетевой топологии (22\%).

По результатам проведения зачета в 2 семестрах был выявлен рост числа студентов, сдавших его с первого раза - примерно на 30\%.

Таким образом, введение новых средств оценки знаний студентов в виде разработанного ФОС позволило ускорить процесс защиты практических работ студентами. Проведенное анкетирование показало результативность введенных подходов, а также выявило их преимущества и недостатки с точки зрения студентов, проходивших обучение по курсу.

Однако, в связи с полным переходом на дистанционное обучение для всех курсов в РТУ МИРЭА, необходимо перевести контрольные мероприятия в новый формат, удобный для преподавателей и студентов $[8,9]$. Первоочередной задачей является разработка решений для объективной оценки знаний студентов в условиях дистанционного обучения, которое бы основывалось на уже имеющихся наработках ФОС с учетом их модернизации. Ниже приводится одно из возможных решений.

Банк тестовых вариантов заданий по каждой практической работе можно организовать в уже имеющейся системе дистанционного обучения РТУ МИРЭА (https:// online-edu.mirea.ru). Имеющийся ФОС предполагается добавить в качестве контрольных мероприятий по курсу, доступ к которым осуществляется на ограниченное время. Как показывает практика, время, достаточное для прохождения тестового задания, равняется 5 минутам. Следовательно, доступ к прохождению тестов должен будет открываться в определенное время и закрывать- ся по истечению 10 минут - это позволит студентам не только пройти тест, но и оставит время на его открытие и загрузку. Кроме того, сами практические работы студенты должны будут отправлять в установленные сроки для проверки на почту преподавателю в соответствии с правилами, указанными на информационном сайте курca [10].

В дополнение к этому, при дистанционном формате проведения зачета преподавателями должны учитываться дополнительные баллы за активность (до 25 баллов). Число баллов за активность начисляется пропорционально числу сданных в установленные сроки практических заданий.

Данный метод планируется внедрить в случае полностью дистанционного формата проведения практических занятий во 2 семестре по курсу «Основы сетевых технологий». Дальнейшее направление работы заключается в формировании обновленного ФОС для 2 семестра в связи с переходом на новую версию образовательной программы CCNA R\&S, а также в четком определении критериев активности студентов в процессе обучения.

\section{Зак^ючение}

В данной статье был представлен анализ результативности контрольных мероприятий по курсу «Основы сетевых технологий» для студентов 3 курса Института ИТ в РТУ МИРЭА. Они были направлены на индивидуализацию практических заданий и включали в себя разработку большого количества тестовых вариантов практического и теоретического характера.

Для 136 студентов курса авторами было проведено анонимное и добровольное анкетирование, позволившее выявить достоинства и недостатки разработанного ФОС, а полученные диаграммы и ответы студентов позволили проанализировать результативность контрольных мероприятий.

Анкетирование показало, что процесс защиты практических работ стал гораздо быстрее, а также уменьшилось влияние субъективизма со стороны преподавателей. Необходимо отметить, что новые методы должны непрерывно совершенствоваться, а ФОС постоянно обновляться, чтобы учитывать возможные изменения программы курса.

Также в статье было рассмотрено решение, позволяющее осуществить перенос контрольных мероприятий в систему дистанционного обучения. Оптимизация существующих методов и внедрение ускоренной самопроверки студентов будут являться направлениями дальнейшей деятельности преподавателей курса «Основы сетевых технологий». 


\section{ЛИТЕРАТУРА}

1. Ефремова Н.Ф. Критериальные требования к фондам оценочных средств // Педагогические измерения. 2016. №1 [Электронный ресурс] - Режим доступа: https://cyberleninka.ru/article/n/kriterialnye-trebovaniya-k-fondam-otsenochnyh-sredstv (дата обращения: 28.10.2020).

2. Thanasis Daradoumis, Joan Manuel Marquès Puig, Marta Arguedas, Laura Calvet Liñan. Analyzing students' perceptions to improve the design of an automated assessment tool in online distributed programming, Computers \& Education, Volume 128, 2019, Pages 159-170 [Электронный ресурс] - Режим доступа: http://www.sciencedirect.com/science/article/pii/S0360131518302665 (дата обращения 09.12.20).

3. Минин М.Г., Муратова Е.А., Михайлова Н.С. Фонд оценочных средств в структуре образовательных программ // Высшее образование в России. 2011. №5 [Электронный ресурс] - Режим доступа: https://cyberleninka.ru/article/n/fond-otsenochnyh-sredstv-v-strukture-obrazovatelnyh-programm (дата обращения: 28.10.2020).

4. Шингарева М.В. Разработка фонда оценочных средств по учебной дисциплине // Агроинженерия. 2016. №6 (76) [Электронный ресурс] - Режим доступа: https://cyberleninka.ru/article/n/razrabotka-fonda-otsenochnyh-sredstv-po-uchebnoy-distsipline (дата обращения: 02.11.2020).

5. Тулинов С.В., Голубкова Е.М., Круглов А.М. Вопросы совершенствования контрольных мероприятий по курсу «Основы сетевых технологий» // Современная наука: актуальные проблемы теории и практики. Серия «Гуманитарные науки» 2020. №12. С. 108-112

6. Cisco Networking Academy [Электронный ресурс] - Режим доступа: https://www.netacad.com/ru (дата обращения: 20.10.20).

7. E. Gonzalez-Mancebo, M.M. Gandolfo-Cano, M.J. Trujillo-Trujillo, E. Mohedano-Vicente, A. Calso, R. Juarez, A. Melendez, P. Morales, F. Pajuelo., Analysis of the effectiveness of training school personnel in the management of food allergy and anaphylaxis, Allergologia et Immunopathologia, Volume 47, Issue 1, 2019, Pages 60-63 [Электронный ресурс] - Режим доступа: http://www.sciencedirect.com/science/article/pii/S0301054618301046 (дата 06ращения 09.12.20).

8. А.У. Кобилов, А.М. Джурабоев. Технологии компьютерного дистанционного обучения // Academic research in educational sciences. 2020. №3 [Электронный ресурс] - Режим доступа: https://cyberleninka.ru/article/n/tehnologii-kompyuternogo-distantsionnogo-obucheniуа (дата 0бращения: 10.12.2020).

9. Новоселова Д.В., Новоселов Д.В. Дистанционное обучение в условиях пандемии // Теория и практика научных исследований: психология, педагогика, экономика и управление. 2020. №3 (11) [Электронный ресурс] - Режим доступа: https://cyberleninka.ru/article/n/distantsionnoe-obuchenie-v-usloviyahpandemii (дата обращения: 10.12.2020).

10. TERMILAB - Интернет-лаборатория [Электронный ресурс] - Режим доступа: https://lms.termilab.ru/ (дата обращения: 1.09.2020).

\footnotetext{
( Т Тулинов Сергей Владимирович (tulinov@mirea.ru), Голубкова Екатерина Михайловна (golubkova@mirea.ru), Круглов Анатолий Михайлович (kruglov@mirea.ru).

Журнал «Современная наука: актуальные проблемы теории и практики»
}

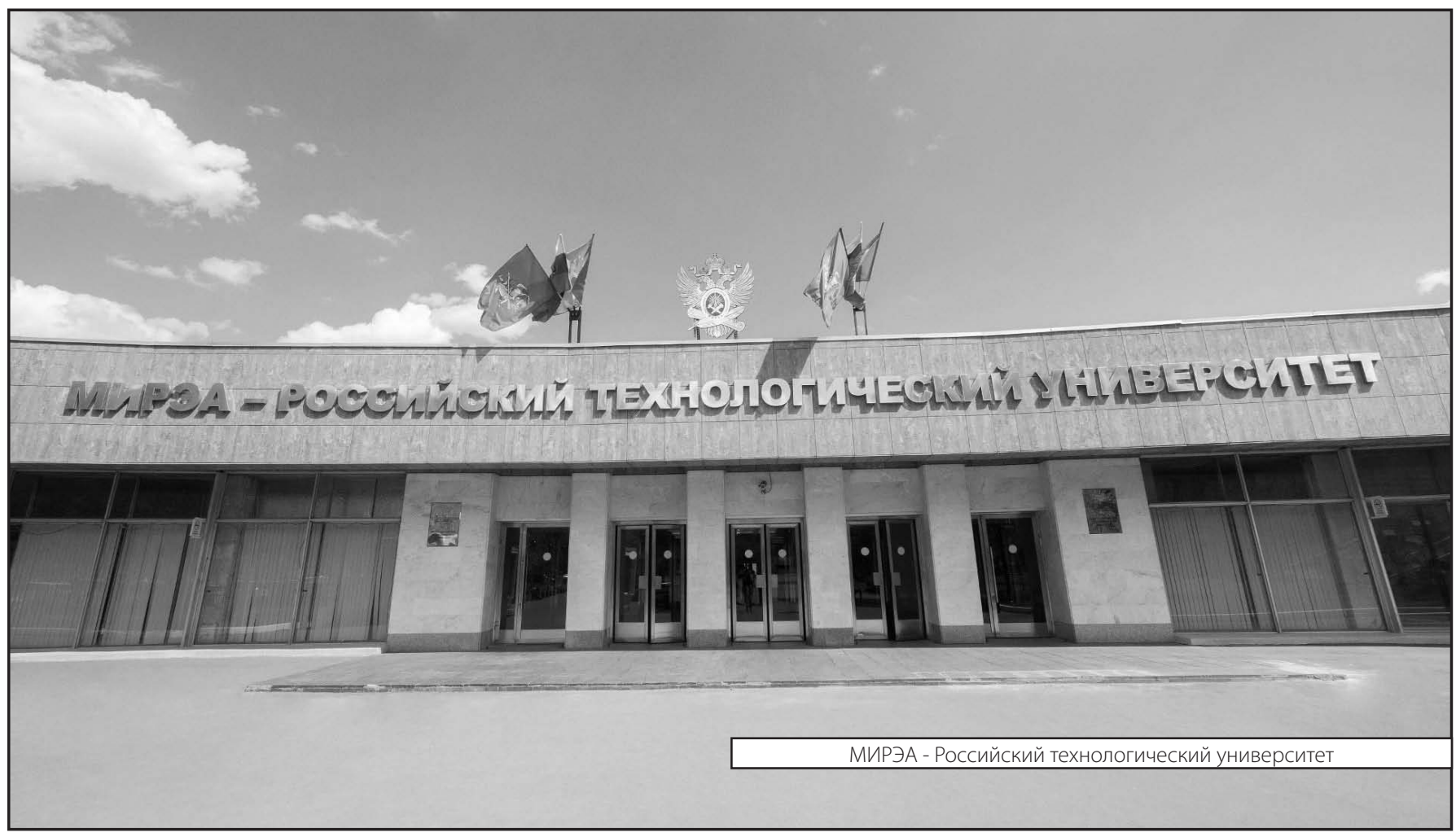

